\title{
EXPONENTIAL STABILITY FOR A NONLINEAR TIMOSHENKO SYSTEM WITH DISTRIBUTED DELAY
}

\section{LAMINE BOUZETTOUTA*, FAHIMA HEBHOUB, KARIMA GHENNAM, SABRINA BENFERDI}

\author{
University of 20 August 1955, Skikda, Algeria \\ ${ }^{*}$ Corresponding author: lami-750000@yahoo.fr
}

\begin{abstract}
This paper is concerned with a nonlinear Timoshenko system modeling clamped thin elastic beams with distributed delay time. The distributed delay is defined on feedback term associated to the equation for rotation angle. Under suitable assumptions on the data, we establish the exponential stability of the system under the usual equal wave speeds assumption.
\end{abstract}

\section{INTRODUCTION}

In this work, we consider the following non linear Timoshenko system with distributed delay,

$$
\left\{\begin{array}{l}
\rho_{1} \varphi_{t t}-k\left(\varphi_{x}+\psi\right)_{x}=0 \\
\rho_{2} \psi_{t t}-b \psi_{x x}+k\left(\varphi_{x}+\psi\right)+\mu_{1} \psi_{t} \\
+\int_{\tau_{1}}^{\tau_{2}} \mu_{2}(s) \psi_{t}(x, t-s) d s+f(\psi)=0
\end{array}\right.
$$

where $t$ denotes the time variable and $x$ the space variable along a beam of length 1 in its equilibrium configuration. Here, $\varphi=\varphi(x, t)$ and $\psi=\psi(x, t)$ denotes the transverse displacement of the beam and the rotation angle of its filament, respectively. The term $\mu_{1} \psi_{t}$ represents a frictional damping and $f(\psi)$ is a forcing term. The coefficients, $\rho_{1}, \rho_{2}, k$ are positive constants represent the density, the polar momentum

Received October $13^{\text {th }}, 2020$; accepted November $5^{\text {th }}, 2020$; published December $4^{\text {th }}, 2020$.

2010 Mathematics Subject Classification. 93D15, 93D20, 35B37, 35B40, 74D05.

Key words and phrases. Timoshenko system, distributed delay time, exponential stability, Lyapunov functional.

(C)2021 Authors retain the copyrights of their papers, and all open access articles are distributed under the terms of the Creative Commons Attribution License. 
of inertia of a cross section, shear modulus respectively, and $b=E I$ where $E$ is the young's modulus of elasticity, $I$ is the moment of inertia cross-section.

System (1.1) is supplemented with the following initial conditions

$$
\begin{aligned}
& \varphi(x, 0)=\varphi_{0}, \varphi_{t}(x, 0)=\varphi_{t}, \psi(x, 0)=\psi_{0}, \psi_{t}(x, 0)=\psi_{1} \\
& \psi_{t}(x,-t)=f_{0}(x, t)
\end{aligned}
$$

and Dirichlet boundary conditions

$$
\varphi(0, t)=\varphi(1, t)=\psi(0, t)=\psi(1, t)
$$

where $x \in(0,1), t \in\left(\tau_{1}, \tau_{2}\right)$. The initial data $\left(\varphi_{0}, \psi_{0}, \varphi_{1}, \psi_{1}, f_{0}\right)$ belongs to a suitable functional spacial.

This type of problems (without delay), has been considered, first in [15] where $\mu_{1}=\mu_{2}=f=0$. The stability of this problems has received much attention in last years, we can find in the literature many results about different stability of Timoshenko systems depending, in particular, on the weights $\mu_{1}$ and $\mu_{2}$ (see [14]) Recently also a great consideration ha been addressed to time delay effects. On such problems, it was showed that a small delay acted on a boundary control, or internal can destabilize a system which is uniformly asymptotically stable in the absence of delays. See for instance $([5])$.

In [13] S. Nicaise and C. Pignotti examined a system of wave equation with initial feedback

$$
\left\{\begin{array}{l}
u_{t t}-u_{x x}+\mu_{0} u_{t}+\int_{\tau_{1}}^{\tau_{2}} a(x) \mu(s) u_{t}(t-s) d s \\
u=0 \quad \text { on } \quad \Gamma_{0}(0, \alpha) \\
\frac{\partial u}{\partial \nu}=0 \quad \text { on } \quad \Gamma_{1}(0, \alpha) \\
u(x, 0)=u_{0}(x) \quad \text { and } \quad u_{t}(x, 0)=u_{1}(x) \quad \text { in } \Omega \\
u_{t}(x,-t)=f_{0}(x,-t) \quad \text { in } \Omega\left(0, \tau_{2}\right)
\end{array}\right.
$$

where $a \in L^{2}(\Omega)$ is a function chosen with some assumptions. They proved that the above system is exponentially stable under the condition

$$
\mu_{0}>\|a\|_{\alpha} \int_{\tau_{1}}^{\tau_{2}} \mu(s) d s
$$

Similarly result was obtained by the authors when the distributed delay acted on the part of boundary. In [11] Mustapha considered a Timoshenko system of thermoelasticity of type III with distributed delay and establish the stability for the case of equal and non equal speeds of wave propagation .Appalara [1] 
investigated a thermo-elastic system of Timoshenko type with second sound and distributed delay

$$
\left\{\begin{array}{l}
\rho_{1} \varphi_{t t}-k\left(\varphi_{x}+\psi\right)_{x}+\gamma_{1} \varphi_{t}+\int_{\tau_{1}}^{\tau_{2}} \gamma_{2}(s) \varphi_{t}(x, t-s)=0 \\
\rho_{2} \psi_{t t}-b \psi_{x x}+k\left(\varphi_{x}+\psi\right)+\delta \theta_{x}=0 \\
\rho_{3} \theta_{t}+q_{x}+\delta \psi_{t x}=0 \\
\tau q_{t}+\beta q+\theta_{x}=0
\end{array}\right.
$$

in $(0,1)(0, \alpha)$, this system is exponentially stable regardless the speeds of wave propagation. The same author studied in [2] a one dimensional Timoshenko system with linear frictional damping and a distributed delay acting on the displacement equation, he showed that dissipation through the frictional damping is strong enough to uniformly stabilize the system. for other results about different types of time delay (discrete and continuos delay) we refer the reader to see $([1-4,8,10])$.

B. Feng and H. L. Pelier [6] considered a following non linear Timoshenko system with constant delay and forcing term:

$$
\left\{\begin{array}{l}
\rho_{1} \varphi_{t t}-k\left(\varphi_{x}+\psi\right)_{x}=0 \\
\rho_{2} \psi_{t t}-b \psi_{x x}+k\left(\varphi_{x}+\psi\right)+\mu_{1} \psi_{t}+\mu_{2}(s) \psi_{t}(x, t-\tau)+f(\psi)=0
\end{array}\right.
$$

and obtained an exponential stability under equal wave speeds.

Recently S. A. Messaoudi, B. Said-Houari [10] established the stability of a thermoelastic Timoshenko system of type III with past history and distributed delay for the cases of equal and non equal speeds of wave propagation respectively.

In the present work, we extend the result of Feng and Pelier, [6] where constant delay is replaced by distributed delay.

\section{Preliminaries}

In this section we present the some assumptions needed later to prove our results. As in [12], we introduce the following new dependent variable

$$
z(x, \rho, s, t)=\psi_{t}(x, t-\rho s), x \in(0,1), \rho \in(0,1), t, s \in\left(\tau_{1}, \tau_{2}\right)
$$

Then, the above variable $z$ satisfies

$$
s z_{t}(x, \rho, s, t)+z_{\rho}(x, \rho, s, t)=0,(x, \rho, s, t) \in(0,1) \times(0,1) \times\left(\tau_{1}, \tau_{2}\right) \times(0,+\infty) .
$$


Therefore, the problem (1.1) is equivalent to

$$
\left\{\begin{array}{l}
\rho_{1} \varphi_{t t}-k\left(\varphi_{x}+\psi\right)_{x}=0, x \in(0,1), t>0 \\
\rho_{2} \psi_{t t}-b \psi_{x x}+k\left(\varphi_{x}+\psi\right)+\mu_{1} \psi_{t} \\
+\int_{\tau_{1}}^{\tau_{2}} \mu_{2}(s) z(x, 1, s, t) d s+f(\psi)=0, x \in(0,1), t>0 \\
s z_{t}(x, \rho, s, t)+z_{\rho}(x, \rho, s, t)=0, \rho \in(0,1), s \in\left(\tau_{1}, \tau_{2}\right), t>0
\end{array}\right.
$$

with the following initial and boundary conditions

$$
\left\{\begin{array}{l}
\varphi(x, 0)=\varphi_{0}, \varphi_{t}(x, 0)=\varphi_{1}, x \in(0,1) \\
\psi(x, 0)=\psi_{0}, \psi_{t}(x, 0)=\psi_{1}, x \in(0,1) \\
z(x, \rho, s, 0)=f_{0}(x, \rho s), x \in(0,1), \rho \in(0,1), s \in\left(0, \tau_{2}\right) \\
\varphi(0, t)=\varphi(1, t)=\psi(0, t)=\psi(1, t)=0, t>0 .
\end{array}\right.
$$

Concerning the weight of the delay, we only assume that

$$
\int_{\tau_{1}}^{\tau_{2}}\left|\mu_{2}(s)\right| d s<\mu_{1}
$$

In addition, we give some hypothesis on the forcing term $f(\psi(x, t))$. We assume that $f: I R \rightarrow I R$ satisfies the following condition

$$
\left|f\left(\psi^{1}\right)-f(\psi 2)\right| \leq k_{0}\left(\left|\psi^{1}\right|^{\theta}-\left|\psi^{2}\right|^{\theta}\right)\left|\psi^{1}-\psi^{2}\right|
$$

for all $\psi^{1}, \psi^{2} \in I R$, where $k_{0}>0, \theta>0$. Also

$$
0 \leq \tilde{f}(\psi) \leq f(\psi) \psi, \text { for all } \psi \in I R,
$$

with

$$
\tilde{f}(y)=\int_{0}^{y} f(s) d s
$$

We introduce the Hilbert space,

$$
\mathcal{H}=H_{0}^{1}(0,1) \times L^{2}(0,1) \times H_{0}^{1}(0,1) \times L^{2}(0,1) \times L^{2}\left((0,1) \times(0,1) \times\left(\tau_{1}, \tau_{2}\right)\right)
$$

For $U=(\varphi, u, \psi, v, z)^{T},(\widetilde{\varphi}, \widetilde{u}, \widetilde{\psi}, \widetilde{v}, \widetilde{z})^{T}$ equipped with the scalar product

$$
\begin{aligned}
\langle u, \widetilde{u}\rangle_{H} & =\int_{0}^{1}\left[\rho_{1} u \widetilde{u}+\rho_{2} v \widetilde{v}+k\left(\varphi_{x}+\psi\right)\left(\widetilde{\varphi}_{x}+\widetilde{\psi}\right)+b \psi_{x} \widetilde{\psi}_{x}\right] d x \\
& +\int_{0}^{1} \int_{\tau_{1}}^{\tau_{2}} s\left|\mu_{2}(s)\right| \int_{0}^{1} z(x, \rho, s, t) \widetilde{z}(x, \rho, s, t) d \rho d s d x .
\end{aligned}
$$

We introduce two new dependent variables $\varphi_{t}=u$ and $\psi_{t}=v$, then the system (2.1)-(2.2) can be written as

$$
\left\{\begin{array}{l}
\frac{\partial U}{\partial t}=A U+F, t>0 \\
U(x, 0)=U^{0}(x)=\left(\varphi^{0}, \varphi^{1}, \psi^{0}, \psi^{1}, f_{0}\right)^{T}
\end{array}\right.
$$


and

$$
\begin{gathered}
A U=\left(\begin{array}{c}
u \\
\frac{k}{\rho_{1}}\left(\varphi_{x x}+\psi_{x}\right) \\
v \\
\frac{b}{\rho_{2}} \psi_{x x}-\frac{k}{\rho_{2}}\left(\varphi_{x}+\psi\right)-\frac{\mu_{1}}{\rho_{2}} v-\frac{\mu_{1}}{\rho_{2}} \int_{\tau_{1}}^{\tau_{2}} \mu_{2}(s) z(x, \rho, s, t) d s \\
-\frac{1}{\tau} z_{\rho}(x, \rho, s, t) \\
F=\left(\begin{array}{c}
0 \\
0 \\
0 \\
\frac{-1}{\rho^{2}} f(\psi) \\
0
\end{array}\right)
\end{array}\right)
\end{gathered}
$$

with the domain

$$
D(A)=\left\{(\varphi, u, \psi, v, z)^{T} \in H: v=z(x, 0, s, t) \text { in }(0,1)\right\}
$$

where

$$
\begin{aligned}
H & =\left(H^{2}(0,1) \cap H_{0}^{1}(0,1)\right) \times H_{0}^{1}(0,1) \times\left(H^{2}(0,1) \cap H_{0}^{1}(0,1)\right) \\
& \times H_{0}^{1}(0,1) \times L^{2}\left((0,1) \times(0,1) \times\left(\tau_{1}, \tau_{2}\right)\right) .
\end{aligned}
$$

Clearly, $D(A)$ is dense in $H$, we have the following existence and uniqueness result (see [6]).

Theorem 2.1. Let $U_{0} \in \mathcal{H}$ and assume that (2.4)-(2.5) and $\mu_{2}<\mu_{1}$ hold. Then, there exists a unique solution $U \in C\left(\mathbb{R}^{+}, \mathcal{H}\right)$ of problem $(2.1)$. Moreover, if $U_{0} \in D(A)$, then $U \in C\left(\mathbb{R}^{+}, D(A)\right) \cap C\left(\mathbb{R}^{+}, \mathcal{H}\right)$.

\section{Stability RESUlt}

In this section, we use the energy method to show that the solution of problem (2.1)-(2.2) decays exponentially, below we shall give the stability result.

Theorem 3.1. Assume that(2.4)-(2.5) and $\mu_{2}<\mu_{1}$ hold. Assume that $\frac{\rho_{1}}{\rho_{2}}=\frac{k}{b}$ also holds. Then, with respect to mild solutions, there exist $\varpi_{1}>0$ and $\varpi_{2}>0$ such that

$$
E(t) \leq \varpi_{1} e^{-\varpi_{2} t}, t \geq 0
$$

To achieve our goal we state and prove the following lemmas.

Lemma 3.1. The energy functional $E(t)$ of problem (2.1)-(2.2), defined by

$$
\begin{aligned}
E(t) & =\frac{1}{2} \int_{0}^{1}\left(\rho_{1} \varphi_{t}^{2}+\rho_{2} \psi_{t}^{2}\right) d x+\frac{1}{2} \int_{0}^{1}\left\{K\left(\varphi_{x}+\psi\right)^{2}+b \psi_{x}^{2}\right\} d x \\
& +\int_{0}^{1} \int_{0}^{1} \int_{\tau_{1}}^{\tau_{2}} s\left|\mu_{2}(s)\right| z(x, \rho, s, t) d s d \rho d x+\int_{0}^{1} \tilde{f}(\psi) d x
\end{aligned}
$$


satisfies

$$
\frac{d E(t)}{d t} \leq-m_{1} \int_{0}^{1} \psi_{t}^{2} d x \leq 0
$$

where $m_{1}=\mu_{1}-\int_{\tau_{1}}^{\tau_{2}}\left|\mu_{2}(s)\right| d s$

Proof. Multiplying the first equation in $(2.1)$ by $\varphi_{t}$, the second equation by $\psi_{t}$, integrating over $(0,1)$ and summing them up we get

$$
\begin{aligned}
& \frac{1}{2} \frac{d}{d t} \int_{0}^{1}\left(\rho_{1} \varphi_{t}^{2}+\rho_{2} \psi_{t}^{2}\right) d x+\frac{1}{2} \frac{d}{d t} \int_{0}^{1}\left\{K\left(\varphi_{x}+\psi\right)^{2}+b \psi_{x}^{2}\right\} d x \\
& =-\mu_{1} \int_{0}^{1} \psi_{t}^{2} d x-\mu_{1} \int_{0}^{1} f(\psi) \psi_{t} d x-\int_{0}^{1} \int_{\tau_{1}}^{\tau_{2}} \psi_{t} \mu_{2}(s) z(x, 1, s, t) d s d x .
\end{aligned}
$$

Multiplying the third equation of (2.1) by $\left|\mu_{2}(s)\right| z(x, \rho, s, t)$ and integrating over $(0,1) \times(0,1) \times\left(\tau_{1}, \tau_{2}\right)$, we obtain

$$
\begin{aligned}
& \frac{1}{2} \frac{d}{d t} \int_{0}^{1} \int_{0}^{1} \int_{\tau_{1}}^{\tau_{2}} s\left|\mu_{2}(s)\right| z^{2}(x, \rho, s, t) d s d \rho d x \\
& +\frac{1}{2} \int_{0}^{1} \int_{\tau_{1}}^{\tau_{2}}\left|\mu_{2}(s)\right| z^{2}(x, 1, s, t) d s d x \\
& -\frac{1}{2} \int_{0}^{1} \int_{\tau_{1}}^{\tau_{2}}\left|\mu_{2}(s)\right| z^{2}(x, 0, s, t) d s d x=0
\end{aligned}
$$

by summing (3.5), (3.4) and using the fact that $z(x, 0, s, t)=\varphi_{t}(x, t)$, we have

$$
\begin{aligned}
\frac{d E(t)}{d t} & =-\left(\mu_{1}-\frac{1}{2} \int_{\tau_{1}}^{\tau_{2}}\left|\mu_{2}(s)\right| d s\right) \int_{0}^{1} \psi_{t}^{2} d x \\
& -\frac{1}{2} \int_{0}^{1} \int_{\tau_{1}}^{\tau_{2}}\left|\mu_{2}(s)\right| z^{2}(x, 1, s, t) d s d x \\
& -\int_{0}^{1} \psi_{t} \int_{\tau_{1}}^{\tau_{2}} \mu_{2}(s) z(x, 1, s, t) d s d x .
\end{aligned}
$$

Now, using Young's inequality, we arrive at

$$
\begin{aligned}
& -\int_{0}^{1} \psi_{t} \int_{\tau_{1}}^{\tau_{2}} \mu_{2}(s) z(x, 1, s, t) d s d x \\
& \leq \frac{1}{2} \int_{\tau_{1}}^{\tau_{2}}\left|\mu_{2}(s)\right| d s \int_{0}^{1} \psi_{t}^{2} d x+\frac{1}{2} \int_{0}^{1} \int_{\tau_{1}}^{\tau_{2}}\left|\mu_{2}(s)\right| z^{2}(x, 1, s, t) d s d x .
\end{aligned}
$$

Inserting (3.7) in (3.6) and using (2.3), we have (3.2) and (3.3). The proof is complete.

Lemma 3.2. Let $(\varphi, \psi, z)$ be the solution of $(2.1)-(2.2)$. Then, the functional

$$
I_{1}(t):=-\int_{0}^{1}\left(\rho_{1} \varphi \varphi_{t}+\rho_{2} \psi \psi_{t}\right) d x-\frac{\mu_{1}}{2} \int_{0}^{1} \psi^{2} d x .
$$


satisfies

$$
\begin{aligned}
\frac{d I_{1}(t)}{d t} & \leq-\int_{0}^{1}\left(\rho_{1} \varphi_{t}^{2}+\rho_{2} \psi_{t}^{2}\right) d x+c_{0} \int_{0}^{1} \psi_{x}^{2} d x+k \int_{0}^{1}\left(\varphi_{x}+\psi\right)^{2} d x \\
& +\frac{\mu_{1}}{4} \int_{0}^{1} \int_{\tau_{1}}^{\tau_{2}}\left|\mu_{2}(s)\right| z^{2}(x, 1, s, t) d s d x,
\end{aligned}
$$

Proof. Differentiating $I_{1}(t)$, we obtain

$$
\begin{aligned}
\frac{d I_{1}(t)}{d t} & =-\rho_{1} \int_{0}^{1} \varphi_{t}^{2} d x-\rho_{1} \int_{0}^{1} \varphi \varphi_{t t} d x-\rho_{2} \int_{0}^{1} \psi_{t}^{2} d x \\
& -\rho_{2} \int_{0}^{1} \psi \psi_{t t} d x-\mu_{1} \int_{0}^{1} \psi \psi_{t} d x
\end{aligned}
$$

and using $(2.1)_{1},(2.1)_{2}$, we get

$$
\begin{aligned}
\frac{d I_{1}(t)}{d t} & =-\rho_{1} \int_{0}^{1} \varphi_{t}^{2} d x-\rho_{2} \int_{0}^{1} \psi_{t}^{2} d x+b \int_{0}^{1} \psi_{x}^{2} d x+k \int_{0}^{1}\left(\varphi_{x}+\psi\right)^{2} d x \\
& +\int_{0}^{1} f(\psi) \psi d x+\int_{0}^{1} \psi \int_{\tau_{1}}^{\tau_{2}} \mu_{2}(s) z(x, 1, s, t) d s d x .
\end{aligned}
$$

Applying Young's and Poincaré inequalities, we have

$$
\begin{aligned}
& \int_{0}^{1} \psi \int_{\tau_{1}}^{\tau_{2}}\left|\mu_{2}(s)\right| z(x, 1, s, t) d s d x \\
& \leq \mu_{1} \int_{0}^{1} \psi_{x}^{2} d x+\frac{\mu_{1}}{4} \int_{0}^{1} \int_{\tau_{1}}^{\tau_{2}}\left|\mu_{2}(s)\right| z^{2}(x, 1, s, t) d s d x \\
& \int_{0}^{1}|f(\psi) \psi| d x \leq \int_{0}^{1}|\psi|^{\theta}|\psi||\psi| d x \\
& \leq\|\psi\|_{2(\theta+1)}^{\theta}\|\psi\|_{2(\theta+1)}\|\psi\| \\
& \leq c_{1} \int_{0}^{1} \psi_{x}^{2} d x
\end{aligned}
$$

By substituting (3.11), (3.12) in (3.10), we obtain (3.9).

Now, let $w$ be the solution of

$$
-w_{x x}=\psi_{x}, \quad w(0)=w(1)=0,
$$

then we get

$$
w(x, t)=-\int_{0}^{x} \psi(y, t) d y+x\left(\int_{0}^{1} \psi(y, t) d y\right) .
$$

We have the following inequalities.

Lemma 3.3. The solution of (3.13) satisfies

$$
\int_{0}^{1} w_{x}^{2} d x \leq \int_{0}^{1} \psi^{2} d x \text { and } \int_{0}^{1} w_{t}^{2} d x \leq \int_{0}^{1} \psi_{t}^{2} d x .
$$


Proof. We multiply equation (3.13) by $w$, integrate by parts and use the Cauchy-Schwarz inequality to obtain

$$
\int_{0}^{1} w_{x}^{2} d x \leq \int_{0}^{1} \psi^{2} d x
$$

Next, we differentiate (3.13) with respect to $t$ and by the same procedure as above, we obtain

$$
\int_{0}^{1} w_{t}^{2} d x \leq \int_{0}^{1} \psi_{t}^{2} d x
$$

This completes the proof of Lemma (3.3).

Lemma 3.4. Let $(\varphi, \psi, z)$ be the solution of (2.1)-(2.2). Then, for any $\varepsilon_{2}>0$, the functional

$$
I_{2}(t):=\int_{0}^{1}\left(\rho_{2} \psi_{t} \psi+\rho_{1} \varphi_{t} w+\frac{\mu_{1}}{2} \psi^{2}\right) d x
$$

satisfies

$$
\begin{aligned}
\frac{d I_{2}(t)}{d t} & \leq-\frac{b}{2} \int_{0}^{1} \psi_{x}^{2} d x+\left(\frac{\rho_{1}}{4 \varepsilon_{2}}+\rho_{2}\right) \int_{0}^{1} \psi_{t}^{2} d x+\rho_{1} \varepsilon_{2} \int_{0}^{1} \varphi_{t}^{2} d x \\
& +\frac{\mu_{1}}{4 \varepsilon_{2}} \int_{0}^{1}\left(\int_{\tau_{1}}^{\tau_{2}}\left|\mu_{2}(s)\right| z^{2}(x, 1, s, t) d s\right) d x-\int_{0}^{1} \tilde{f}(\psi) d x
\end{aligned}
$$

Proof. By differentiation $I_{2}(t)$, we obtain and by using $(2.1)_{1},(2.1)_{2}$, we have

$$
\begin{aligned}
\frac{d I_{2}(t)}{d t} & =\rho_{2} \int_{0}^{1} \psi_{t}^{2} d x-b \int_{0}^{1} \psi_{x}^{2} d x+\rho_{1} \int_{0}^{1} \varphi_{t} w_{t} d x-k \int_{0}^{1} \psi^{2} d x+k \int_{0}^{1} w_{x}^{2} d x \\
& -\int_{0}^{1} f(\psi) \psi d x-\int_{0}^{1} \psi\left(\int_{\tau_{1}}^{\tau_{2}} \mu_{2}(s) z(x, 1, s, t) d s\right) d x
\end{aligned}
$$

Using Young's inequality and (3.15), we have

$$
\begin{aligned}
\rho_{1} \int_{0}^{1} \varphi_{t} w_{t} d x & \leq \rho_{1} \varepsilon_{2} \int_{0}^{1} \varphi_{t}^{2} d x+\frac{\rho_{1}}{4 \varepsilon_{2}} \int_{0}^{1} w_{t}^{2} d x \\
& \leq \rho_{1} \varepsilon_{2} \int_{0}^{1} \varphi_{t}^{2} d x+\frac{\rho_{1}}{4 \varepsilon_{2}} \int_{0}^{1} \psi_{t}^{2} d x
\end{aligned}
$$

Using Young's, Cauchy-Schwarz, Poincaré inequalities, we get

$$
\begin{aligned}
& -\int_{0}^{1} \psi\left(\int_{\tau_{1}}^{\tau_{2}}\left|\mu_{2}(s) z(x, 1, s, t)\right| d s\right) d x \\
& \leq \delta_{1} \int_{0}^{1} \psi_{x}^{2} d x+\frac{\mu_{1}}{4 \delta_{1}} \int_{0}^{1}\left(\int_{\tau_{1}}^{\tau_{2}}\left|\mu_{2}(s)\right| z^{2}(x, 1, s, t) d s\right) d x
\end{aligned}
$$

Cauchy-Schwarz and Poincaré's inequalities, give

$$
\begin{aligned}
\int_{0}^{1}|f(\psi) \psi| d x & \leq \int_{0}^{1}|\psi|^{\theta}|\psi||\psi| d x \\
& \leq\|\psi\|_{2(\theta+1)}^{\theta}\|\psi\|_{2(\theta+1)}\|\psi\| \\
& \leq c_{1} \int_{0}^{1} \psi_{x}^{2} d x
\end{aligned}
$$


By substituting (3.19), (3.20), (3.21) in (3.18), recalling (3.14), (3.15), (2.5) and letting $\delta_{1}=\frac{b}{2}$, we obtain (3.17). The proof is now complete.

Lemma 3.5. Let $(\varphi, \psi, z)$ be the solution of (2.1)-(2.2). Then, the functional

$$
I_{3}(t):=\rho_{2} \int_{0}^{1} \psi_{t}\left(\varphi_{x}+\psi\right)+\rho_{2} \int_{0}^{1} \psi_{x} \varphi_{t} d x
$$

satisfies

$$
\begin{aligned}
\frac{d I_{3}(t)}{d t} & \leq b\left[\psi_{x} \varphi_{x}\right]_{0}^{1} d x+\left(\rho_{2}+\frac{\mu_{1}^{2}}{k}\right) \int_{0}^{1} \psi_{t}^{2} d x-\frac{k}{4} \int_{0}^{1}\left(\varphi_{x}+\psi\right)^{2} d x \\
& +c_{1} \int_{0}^{1} \psi_{x}^{2} d x+\frac{\mu_{1}}{k} \int_{0}^{1} \int_{\tau_{1}}^{\tau_{2}}\left|\mu_{2}(s)\right| z^{2}(x, 1, s, t) d s d x-\int_{0}^{1} \tilde{f}(\psi) d x \\
& +\left(\frac{\rho_{2} k-\rho_{1} b}{\rho_{1}}\right) \int_{0}^{1} \psi_{x}\left(\varphi_{x}+\psi\right)_{x} d x
\end{aligned}
$$

where $c_{1}$ is a positive constant.

Proof. By differentiation $I_{3}(t)$ and using $(2.1)_{1},(2.1)_{2}$, we obtain

$$
\begin{aligned}
\frac{d I_{3}(t)}{d t} & =b\left[\psi_{x} \varphi_{x}\right]_{0}^{1}+\rho_{2} \int_{0}^{1} \psi_{t}^{2} d x-k \int_{0}^{1}\left(\varphi_{x}+\psi\right)^{2} d x-\mu_{1} \int_{0}^{1} \psi_{t}\left(\varphi_{x}+\psi\right) d x \\
& -\int_{0}^{1} \int_{\tau_{1}}^{\tau_{2}} \mu_{2}(s)\left(\varphi_{x}+\psi\right) z(x, 1, s, t) d s d x-\int_{0}^{1} f(\psi)\left(\varphi_{x}+\psi\right) d x
\end{aligned}
$$

By using Young's inequality, we have

$$
\mu_{1} \int_{0}^{1}\left|\psi_{t}\left(\varphi_{x}+\psi\right)\right| d x \leq \frac{k}{4} \int_{0}^{1}\left(\varphi_{x}+\psi\right)^{2} d x+\frac{\mu_{1}^{2}}{k} \int_{0}^{1} \psi_{t}^{2} d x
$$

Using Young's and Cauchy Schwarz inequalities, we get

$$
\begin{aligned}
& \int_{0}^{1}\left(\varphi_{x}+\psi\right) \int_{\tau_{1}}^{\tau_{2}}\left|\mu_{2}(s) z(x, 1, s, t)\right| d s d x \\
& \leq \frac{k}{4} \int_{0}^{1}\left(\varphi_{x}+\psi\right)^{2} d x+\frac{\mu_{1}^{2}}{k} \int_{0}^{1} \int_{\tau_{1}}^{\tau_{2}}\left|\mu_{2}(s)\right| z^{2}(x, 1, s, t) d s d x
\end{aligned}
$$

Young's, Cauchy Schwarz and Poincaré inequalities lead to

$$
\begin{aligned}
\int_{0}^{1} f(\psi) \varphi_{x} d x & \leq\left\|\varphi_{x}\right\|\|\psi\|_{2(\theta+1)}^{\theta}\|\psi\|_{2(\theta+1)} \\
& \leq \frac{\delta_{0}}{2 b^{2}} \int_{0}^{1} \varphi_{x}^{2} d x+\frac{b^{2}}{2 \delta_{0} \lambda_{1}} \int_{0}^{1} \psi_{x}^{2} d x \\
& \leq \frac{\delta_{0}}{2 b^{2}} \int_{0}^{1}\left(\varphi_{x}+\psi\right)^{2} d x+\frac{\delta_{0}}{2 b^{2}} \int_{0}^{1} \psi^{2} d x+\frac{b^{2}}{2 \delta_{0} \lambda_{1}} \int_{0}^{1} \psi_{x}^{2} d x \\
& \leq \frac{\delta_{0}}{2 b^{2}} \int_{0}^{1}\left(\varphi_{x}+\psi\right)^{2} d x+\left(\frac{\delta_{0}}{2 \lambda_{1} b^{2}}+\frac{b^{2}}{2 \delta_{0} \lambda_{1}}\right) \int_{0}^{1} \psi_{x}^{2} d x
\end{aligned}
$$

Inserting (3.25)-(3.27) in (3.24) and letting $\delta_{0}=\frac{1}{2} k b^{2}$, we obtain (3.23). 
Next, in order to handle the boundary terms, appearing in (3.23), we define the function

$$
q(x)=-4 x+2, \quad x \in(0,1)
$$

So, we have the following result.

Lemma 3.6. Let $(\varphi, \psi, z)$ be the solution of (2.1)-(2.2), then for any $\varepsilon_{1}>0$, the following estimate holds

$$
\begin{aligned}
& b\left[\psi_{x} \varphi_{x}\right]_{0}^{1} \leq-\frac{b \rho_{2}}{4 \varepsilon_{1}} \frac{d}{d t} \int_{0}^{1} q \psi_{t} \psi_{x} d x-\frac{\rho_{1} \varepsilon_{1}}{k} \frac{d}{d t} \int_{0}^{1} q \varphi_{t} \varphi_{x} d x+3 \varepsilon_{1} \int_{0}^{1} \varphi_{x}^{2} d x \\
& +\left(\frac{2 \rho_{1} \varepsilon_{1}}{k}+\frac{b \rho_{2}}{2 \varepsilon_{1}}\right) \int_{0}^{1} \psi_{t}^{2} d x+\left(\frac{k^{2} \varepsilon_{1}^{2}}{4}+\frac{\varepsilon_{1}}{4 b^{2}}\right) \int_{0}^{1}\left(\varphi_{x}+\psi\right)^{2} d x \\
& +\frac{b}{4 \varepsilon_{1}} \int_{0}^{1} \int_{\tau_{1}}^{\tau_{2}}\left|\mu_{2}(s)\right| z^{2}(x, 1, s, t) d x \\
& +\left(\frac{b^{2}}{2 \varepsilon_{1}^{2}}+\frac{1}{4 \lambda_{1} b^{2}}+\frac{b^{2}}{8 \varepsilon_{1}^{2} \lambda_{1}}+\frac{\mu_{1} b}{4 \varepsilon_{1}}+\frac{b^{2}}{4 \varepsilon_{1}^{3}}+\varepsilon_{1}\right) \int_{0}^{1} \psi_{x}^{2} d x
\end{aligned}
$$

Proof. By using Young's inequality, we easily see that, for $\varepsilon_{1}>0$,

$$
b\left[\psi_{x} \varphi_{x}\right]_{0}^{1} \leq \varepsilon_{1}\left[\varphi_{x}^{2}(1)+\varphi_{x}^{2}(0)\right]+\frac{b^{2}}{4 \varepsilon_{1}}\left[\psi_{x}^{2}(1)+\psi_{x}^{2}(0)\right],
$$

we need the following fact

$$
\frac{d}{d t} \int_{0}^{1} b \rho_{2} q \psi_{t} \psi_{x} d x=b \rho_{2} \int_{0}^{1} q \psi_{t t} \psi_{x} d x+b \rho_{2} \int_{0}^{1} q \psi_{t} \psi_{x t} d x
$$

On the other hand

$$
\begin{aligned}
b \rho_{2} \int_{0}^{1} q \psi_{t t} \psi_{x} d x & =b^{2} \int_{0}^{1} q \psi_{x x} \psi_{x} d x-k b \int_{0}^{1} q\left(\varphi_{t}+\psi\right) \psi_{x} d x \\
& -b \int_{0}^{1} \int_{\tau_{1}}^{\tau_{2}} q \psi_{x} \mu_{2}(s) z(x, 1, s, t) d s d x-b \int_{0}^{1} q f(\psi) \varphi_{x} d x \\
& \leq-b^{2}\left[\psi_{x}^{2}(1)+\psi_{x}^{2}(0)\right]+2 b^{2} \int_{0}^{1} \psi_{x}^{2} d x+\left(k^{2} \varepsilon^{2}+\frac{\varepsilon}{b^{2}}\right) \int_{0}^{1}\left(\varphi_{x}+\psi\right)^{2} d x \\
& +\left(\frac{b^{2}}{\varepsilon^{2}}+\frac{\varepsilon}{2 \lambda_{1} b^{2}}+\frac{b^{2}}{2 \varepsilon \lambda_{1}}+\mu_{1} b\right) \int_{0}^{1} \psi_{x}^{2} d x \\
& +b \int_{0}^{1} \int_{\tau_{1}}^{\tau_{2}}\left|\mu_{2}(s)\right| z^{2}(x, 1, s, t) d s d x .
\end{aligned}
$$

Therefore

$$
b \rho_{2} \int_{0}^{1} q \psi_{t} \psi_{x t} d x=2 \rho_{2} b \int_{0}^{1} \psi_{t}^{2} d x
$$

Similarly

$$
\begin{aligned}
\frac{d}{d t} \int_{0}^{1} \rho_{1} q \varphi_{t} \varphi_{x} d x & =\int_{0}^{1} q\left(\varphi_{t}+\psi\right) \varphi_{x} d x+\int_{0}^{1} \rho_{1} q \varphi_{t} \varphi_{x t} d x \\
& \leq-k\left[\varphi_{x}^{2}(1)+\varphi_{x}^{2}(0)\right]+3 k \int_{0}^{1} \varphi_{x}^{2} d x \\
& +k \int_{0}^{1} \psi_{x}^{2} d x+2 \rho_{1} \int_{0}^{1} \psi_{t}^{2} d x
\end{aligned}
$$


which, along with (3.29)-(3.30), gives us (3.28). The proof is now complete.

Lemma 3.7. Let $(\varphi, \psi, z)$ be the solution of (2.1)-(2.2). Then, for $\eta_{1}>0$, the functional

$$
F_{4}(t)=\int_{0}^{1} \int_{0}^{1} \int_{\tau_{1}}^{\tau_{2}} s e^{-s \rho}\left|\mu_{2}(s)\right| z^{2}(x, \rho, s, t) d s d \rho d x
$$

satisfies

$$
\begin{aligned}
F_{4}^{\prime}(t) & \leq-\eta_{1} \int_{0}^{1} \int_{0}^{1} \int_{\tau_{1}}^{\tau_{2}} s\left|\mu_{2}(s)\right| z^{2}(x, \rho, s, t) d s d \rho d x \\
& -\eta_{1} \int_{0}^{1} \int_{\tau_{1}}^{\tau_{2}}\left|\mu_{2}(s)\right| z^{2}(x, 1, s, t) d s d x+\mu_{1} \int_{0}^{1} \psi_{t}^{2} d x
\end{aligned}
$$

Proof. Differentiating $F_{4}(t)$ and using $(2.1)_{3}$, we obtain

$$
\begin{aligned}
F_{4}^{\prime}(t) & =-2 \int_{0}^{1} \int_{0}^{1} \int_{\tau_{1}}^{\tau_{2}} e^{-s \rho}\left|\mu_{2}(s)\right| z(x, \rho, s, t) z_{\rho}(x, \rho, s, t) d s d \rho d x \\
& =-\int_{0}^{1} \int_{0}^{1} \int_{\tau_{1}}^{\tau_{2}} e^{-s \rho}\left|\mu_{2}(s)\right| \frac{\partial}{\partial \rho}\left[z^{2}(x, \rho, s, t)\right] d s d \rho d x
\end{aligned}
$$

Integration by parts gives and using the fact that $z(x, 0, s, t)=\psi_{t}$ and $e^{-s} \leq e^{-s \rho} \leq 1$, we get for all $\rho \in[0,1]$

$$
\begin{aligned}
F_{4}^{\prime}(t) & \leq-\int_{0}^{1} \int_{\tau_{1}}^{\tau_{2}} e^{-s}\left|\mu_{2}(s)\right| z^{2}(x, 1, s, t) d s d x+\int_{\tau_{1}}^{\tau_{2}}\left|\mu_{2}(s)\right| d s \int_{0}^{1} \psi_{t}^{2} d x \\
& -\int_{0}^{1} \int_{0}^{1} \int_{\tau_{1}}^{\tau_{2}} s e^{-s}\left|\mu_{2}(s)\right| z^{2}(x, \rho, s, t) d s d \rho d x .
\end{aligned}
$$

Since $-e^{-s}$ is an increasing function, we have $-e^{-s} \leq-e^{-\tau_{2}}$ for all $s \in\left[\tau_{1}, \tau_{2}\right]$. Finally, setting $\eta_{1}=$ $e^{-\tau_{2}}$ and recalling (2.3), we obtain (3.32).

Now, we define the Lyapunov functional $L(t)$ by

$$
L(t):=N E(t)+\frac{1}{8} I_{1}(t)+N_{1} I_{2}(t)+I_{3}(t)+N_{2} I_{4}(t),
$$

where $N_{1}, N_{2}$ and $N$ are positive constants.

Lemma 3.8. Let $(\varphi, \psi, z)$ be the solution of $(2.1)-(2.2)$. Then, there exists two positive constants $\beta_{1}$ and $\beta_{2}$ such that the Lyapunov functional $L(t)$ satisfies

$$
\beta_{1} E(t) \leq L(t) \leq \beta_{2} E(t), \quad \forall t \geq 0,
$$

and

$$
L^{\prime}(t) \leq-\lambda_{1} E(t)+\left(\frac{\rho_{2} k-\rho_{1} b}{\rho_{1}}\right) \int_{0}^{1} \psi_{x}\left(\varphi_{x}+\psi\right)_{x} d x
$$


Proof. Let

$$
L(t):=N E(t)+\frac{1}{8} I_{1}(t)+N_{1} I_{2}(t)+I_{3}(t)+N_{2} I_{4}(t),
$$

then

$$
\begin{aligned}
|L(t)-N E(t)| & \leq \frac{\rho_{1}}{8} \int_{0}^{1}\left|\varphi \varphi_{t}\right| d x+\frac{\rho_{2}}{8} \int_{0}^{1}\left|\psi \psi_{t}\right| d x+\frac{\mu_{1}}{16} \int_{0}^{1} \psi^{2} d x \\
& +N_{1} \rho_{2} \int_{0}^{1}\left|\psi_{t} \psi\right| d x+N_{1} \rho_{1} \int_{0}^{1}\left|\varphi_{t} w\right| d x+N_{1} \frac{\mu_{1}}{2} \int_{0}^{1} \psi^{2} d x \\
& +\rho_{2} \int_{0}^{1}\left|\psi_{t}\left(\varphi_{x}+\psi\right)\right| d x+\rho_{2} \int_{0}^{1}\left|\psi_{x} \varphi_{t}\right| d x \\
& +N_{2} \int_{0}^{1} \int_{0}^{1} \int_{\tau_{1}}^{\tau_{2}} s e^{-s \rho}\left|\mu_{2}(s)\right| z^{2}(x, \rho, s, t) d s d \rho d x
\end{aligned}
$$

Exploiting Young's, Poincaré and Cauchy-Schwarz inequalities, we obtain

$$
\begin{aligned}
|L(t)-N E(t)| & \leq C \int_{0}^{1}\left(\psi_{x}^{2}+\psi_{t}^{2}+\varphi_{t}^{2}+\left(\varphi_{x}+\psi\right)^{2}+\int_{0}^{1} \int_{\tau_{1}}^{\tau_{2}} s\left|\mu_{2}(s)\right| z^{2}(x, 1, s, t) d s d \rho\right) d x \\
& +\int_{0}^{1} \tilde{f}(\psi) d x \\
& \leq C E(t)
\end{aligned}
$$

Now, combining (3.3), (3.9), (3.17), (3.23) and (3.32), we get

By differentiating $L(t)$, exploiting (3.3), (3.9), (3.17), (3.23), (3.28), (3.32) and setting $\varepsilon_{2}=\frac{\rho_{1}}{16 N_{1}}$, we get

$$
\begin{aligned}
\frac{d L(t)}{d t} & =-\left(N m_{1}-N_{1}\left(4 N_{1}+\rho_{2}\right)-N_{2} \mu_{1}-\left(\rho_{2}+\frac{\mu_{1}^{2}}{k}\right)-\left(\frac{2 \rho_{1} \varepsilon_{1}}{k}+\frac{b \rho_{2}}{2 \varepsilon_{1}}\right)+\rho_{2}\right) \int_{0}^{1} \psi_{t}^{2} d x \\
- & \left(\frac{b}{2} N_{1}-\frac{c_{0}}{8}-\left(\frac{b^{2}}{2 \varepsilon_{1}^{2}}+\frac{1}{4 b^{2}}+\frac{b^{2}}{8 \varepsilon_{1}^{2}}+\frac{\mu_{1} b}{4 \varepsilon_{1}}+\frac{b^{2}}{4 \varepsilon_{1}^{3}}+\varepsilon_{1}\right)\right) \int_{0}^{1} \psi_{x}^{2} d x \\
& -\left(\frac{k}{8}-\varepsilon_{1}\left(k^{2} \varepsilon_{1}+\frac{1}{b^{2}}\right)\right) \int_{0}^{1}\left(\varphi_{x}+\psi\right)^{2} d x \\
& -N_{2} \beta \int_{0}^{1} \int_{0}^{1} \int_{\tau_{1}}^{\tau_{2}} s\left|\mu_{2}(s)\right| z^{2}(x, \rho, s, t) d s d \rho d x \\
& -\left(N_{2} \beta-4 N_{1}^{2} \mu_{1}-\frac{\mu_{1}}{32}-\frac{\mu_{1}}{k}-\frac{b}{4 \varepsilon_{1}}\right) \int_{0}^{1} \int_{\tau_{1}}^{\tau_{2}}\left|\mu_{2}(s)\right| z^{2}(x, 1, s, t) d s d x \\
& -\frac{\rho_{1}}{16} \int_{0}^{1} \varphi_{t}^{2} d x-\left(N_{1}+1\right) \int_{0}^{1} \tilde{f}(\psi) d x,+\left(\frac{\rho_{2} k-\rho_{1} b}{\rho_{1}}\right) \int_{0}^{1} \psi_{x}\left(\varphi_{x}+\psi\right)_{x} d x .
\end{aligned}
$$

First, we choose $\varepsilon_{1}$ small enough such that

$$
\frac{k}{8}-\varepsilon_{1}\left(k^{2} \varepsilon_{1}+\frac{1}{b^{2}}\right)>0
$$

After, we take $N_{1}$ large so that

$$
\frac{b}{2} N_{1}-\frac{c_{0}}{8}-\left(\frac{b^{2}}{2 \varepsilon_{1}^{2}}+\frac{1}{4 b^{2}}+\frac{b^{2}}{8 \varepsilon_{1}^{2}}+\frac{\mu_{1} b}{4 \varepsilon_{1}}+\frac{b^{2}}{4 \varepsilon_{1}^{3}}+\varepsilon_{1}>0 .\right.
$$


Then, we select $N_{2}$ large to satisfies

$$
N_{2} \beta-4 N_{1}^{2} \mu_{1}-\frac{\mu_{1}}{32}-\frac{\mu_{1}}{k}-\frac{b}{4 \varepsilon_{1}}>0 .
$$

By finally choose $N$ large enough (even larger so that 1.1 remains valid) such that

$$
N m_{1}-N_{1}\left(\frac{\rho_{1}}{4 \varepsilon_{2}}+\rho_{2}\right)-N_{2} \mu_{1}-\left(\rho_{2}+\frac{\mu_{1}^{2}}{k}\right)-\left(\frac{2 \rho_{1} \varepsilon_{1}}{k}+\frac{b \rho_{2}}{2 \varepsilon_{1}}\right)+\rho_{2}>0,
$$

we obtain (3.35). The proof is complete.

Acknowledgments: The authors wish to thank deeply the anonymous referee for his/her useful remarks and his/her careful reading of the proofs presented in this paper.

Conflicts of Interest: The author(s) declare that there are no conflicts of interest regarding the publication of this paper.

\section{REFERENCES}

[1] T.A. Apalara, Well-posedness and exponential stability for a linear damped Timoshenko system with second sound and internal distributed delay. Electron. J. Differ. Equ. 2014 (2014), 254.

[2] T.A. Apalara, Uniform decay in weakly dissipative timoshenko system with internal distributed delay feedbacks, Acta Math. Sci. 36 (2016), 815-830.

[3] L. Bouzettouta, D. Abdelhak, Exponential stabilization of the full von Kármán beam by a thermal effect and a frictional damping and distributed delay, J. Math. Phys. 60 (2019), 041506.

[4] L. Bouzettouta, S. Zitouni, Kh. Zennir. and H. Sissaoui, Stability of Bresse system with internal distributed delay. J. Math. Comput. Sci. 7(1) (2017), 92-118.

[5] R. Datko, J. Lagnese and M. P. Polis, An example on the effect of time delays in boundary feedback stabilization of wave equations. SIAM J. Control Optim. 24(1) (1986), 152-156.

[6] B.W. Feng and M. L. Pelicer, Global existence and exponential stability for a nonlinear Timoshenko system with delay. Bound. Value Probl. 2015 (2015), Article ID 206.

[7] H.E. Khochemane, A. Djebabla, S. Zitouni, L. Bouzettouta, Well-posedness and general decay of a nonlinear damping porous-elastic system with infinite memory, J. Math. Phys. 61 (2020), 021505.

[8] H.E. Khochemane, L. Bouzettouta, A. Guerouah, Exponential decay and well-posedness for a one-dimensional porouselastic system with distributed delay, Appl. Anal. (2019), 1-15. https://doi.org/10.1080/00036811.2019.1703958.

[9] H. E. Khochemane, S. Zitouni and L. Bouzettouta, Stability result for a nonlinear damping porous-elastic system with delay term, Nonlinear studies, 27(2) (2020), 1-17.

[10] S. A. Messaoudi and B. Said-Houari, Energy decay in a Timoshenko-type system for thermoelasticity of type III with distributed delay and past history, Electron. J. Differ. Equ. 2018 (2018), 75.

[11] M.I. Mustafa, A uniform stability result for thermoelasticity of type III with boundary distributed delay, J. Math. Anal. Appl. 415 (2014), 148-158.

[12] S. Nicaise and C. Pignotti, Stability and instability results of the wave equation with a delay term in the boundary or internal feedbacks. SIAM J. Control Optim. 45(5) (2006), 1561-1585.

[13] S. Nicaise and C. Pignotti, Interior feedback stabilization of wave equations with time dependent delay. Electron. J. Differ. Equ. 2011 (2011), 41. 
[14] C. Pignotti, A note on stabilization of locally damped wave equations with time delay, Syst. Control Lett. 61 (2012), 92-97.

[15] S. P. Timoshenko, On the correction for shear of the differential equation for transverse vibrations of prismatic bars. Phil. Mag. Ser. 6(41) (1921), 744-746.

[16] S. Zitouni, L. Bouzettouta, Kh. Zennir and D. Ouchenane, Exponential decay of thermo-elastic Bresse system with distributed delay term, Hacettepe J. Math. Stat. 47(5) (2018), 1216-1230. 